\title{
Elements of the design and analysis of quantum-dot intermediate band solar cells
}

\author{
A. Martí ，E. Antolín ， E. Cánovas , N. López , P.G. Linares ，A. Luque , C.R. Stanley ， \\ C.D. Farmer \\ Instituto de Energía Solar, UPM, ETSIT de Madrid, Ciudad Universitaria sn, 28040 Madrid, Spain \\ Department of Electronics and Electrical Engineering, University of Glasgow, Glasgow G12 8QQ, UK
}

\begin{abstract}
We have demonstrated recently that two below bandgap energy photons can lead to the creation of one electron-hole pair in a quantum-dot intermediate band solar cell (QD-IBSC). To be effective, the devices used in the experiments were designed to a) half-fill the intermediate band with electrons; b) to allocate the quantum dots in a flat-band potential region, and c) to prevent tunnelling from the $\mathrm{n}$ emitter into the intermediate band. QD-IBSCs have also shown degradation in their open-circuit voltage when compared with their counterparts without quantum dots. This loss is due to the presence of the intermediate band (IB) together with the incapacity of the quantum dots to absorb sufficient below bandgap light as to contribute significantly to the photogenerated current. It is predicted, nevertheless, that this voltage loss will diminish if concentration light is used leading to devices with efficiency higher than single gap solar cells. A circuit model that includes additional recombination levels to the ones introduced by the IB is described to support this discussion.
\end{abstract}

Keywords: Quantum dots; Intermediate band; Photovoltaics; Solar cells; Solar concentration; Novel concepts; InAs; GaAs; Efficiency; Circuit model

\section{Introduction}

The intermediate band solar cell is a novel type of solar cell conceived to use the energy of below bandgap energy photons. To this end, it requires the existence of an intermediate band (IB) located within the semiconductor bandgap (Fig. 1). This band divides the total bandgap of the semiconductor, $E_{\mathrm{G}}$, into two sub-bandgaps, $E_{\mathrm{L}}$ and $E_{\mathrm{H}}$. Thanks to this band, two below bandgap energy photons, as those labelled "1" and "2" in Fig. 1, can create one electron-hole pair by pumping an electron from the valence band (VB) to the intermediate band (photon "1") and an electron from the IB to the conduction band (CB) (photon "2"). To this end, the IB should be half-filled with electrons in order to provide both empty states to receive electrons from the VB as well as electrons to supply to the CB. It is also worthwhile noticing that the creation of an electronhole pair through the IB does not imply a phenomenon of simultaneous collision between three particles (two photons and one electron) since the electron pumped from the VB to the IB does not have to be the same electron promoted by the second photon to the CB. This electron-hole pair adds to the ones conventionally generated by the absorption of a single photon (photon " 3 ") creating a transition from the VB to the CB.

On the other hand, carrier relaxation within the bands is assumed to be a much faster process than carrier recombination between bands. This leads to the assumption that the carrier concentration in each band is described by its quasi-Fermi level ( $E_{\mathrm{F}, \mathrm{C}}, E_{\mathrm{F}, \mathrm{V}}$ and $E_{\mathrm{F}, \mathrm{I}}$ for the $\mathrm{CB}, \mathrm{VB}$ and IB respectively). From the plot in Fig. 1 it can be understood that, since the output voltage of the cell, $V$, is related to the quasi-Fermi level split by $e V=E_{\mathrm{F}, \mathrm{C}}-E_{\mathrm{F}, \mathrm{V}}$, with $e$ being the electron charge, the output voltage of the cell is still limited by the total bandgap $E_{\mathrm{G}}$ and not by $E_{\mathrm{L}}$ or $E_{\mathrm{H}}$.

The concepts expressed above are sufficient to follow the work that will be described in this paper.

The existence of IB materials has been predicted to exist in several systems, as for example $\mathrm{Ti}_{x} \mathrm{Ga}_{1-x} \mathrm{P}$ ite-type semiconductors transition metal-doped chalcopyrand II-VI compounds doped with 


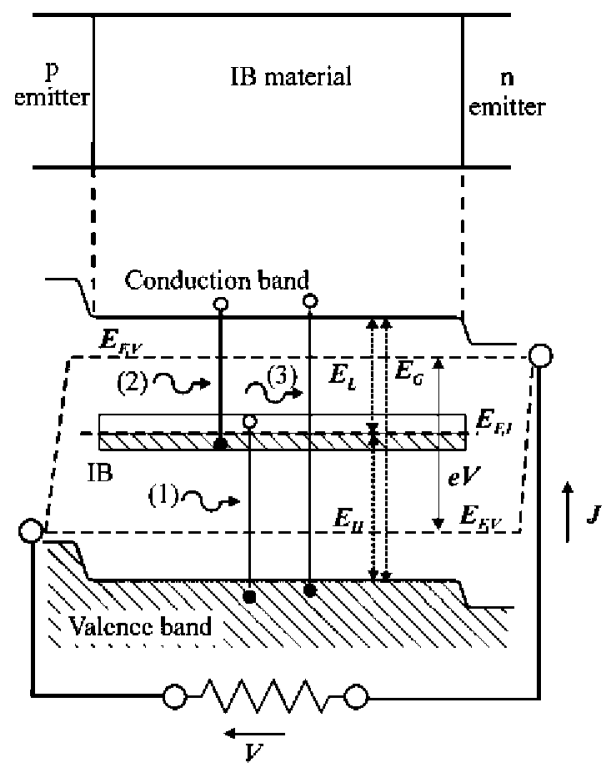

Fig. 1. Simplified bandgap diagram under bias of an intermediate band solar cell indicating several magnitudes of interest such as quasi-Fermi energy levels $\left(E_{\mathrm{F}, \mathrm{C}}\right.$, $E_{\mathrm{F}, \mathrm{I}}$ and $\left.E_{\mathrm{F}, \mathrm{V}}\right)$ and bandgaps $\left(E_{\mathrm{L}}, E_{\mathrm{H}}\right.$ and $E_{\mathrm{G}}$ ). The top figure shows the correspondence of the bandgap diagram with the layer structure.

$\mathrm{Cr} \quad$ Intermediate band materials have been synthetised employing diluted II-VI oxide semiconductors and $\mathrm{GaN}_{x} \mathrm{As}_{1-y} \mathrm{P}_{y}$ alloys In the latest two examples, the formation of the IB is explained on the basis of the band anticrossing model

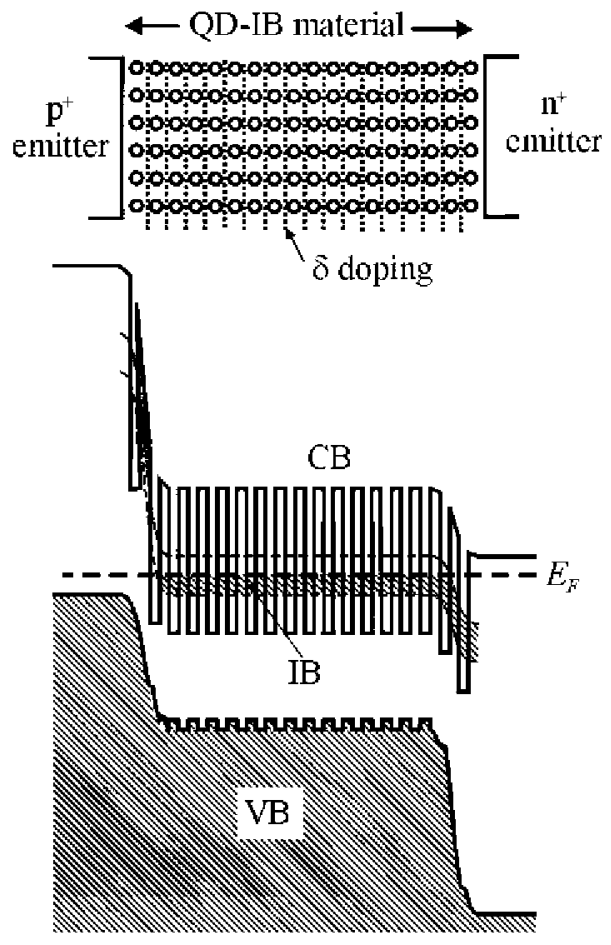

Fig. 2. Simplified bandgap diagram of a quantum-dot intermediate band solar cell in equilibrium. The $\delta$-doping is introduced at the barrier region to half-fill the IB with electrons.

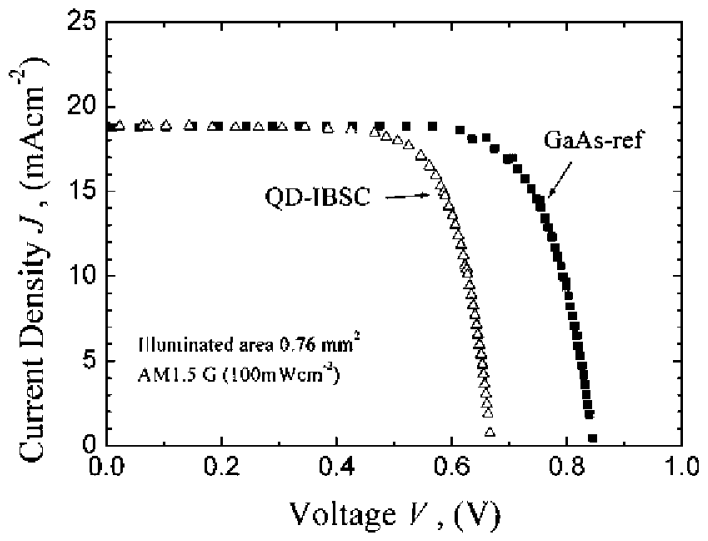

Fig. 3. Typical current-voltage characteristic of a QD-IBSC compared with a reference GaAs cell consisting of the same structure but without quantum dots $(300 \mathrm{~K})$.

Quantum dots (QDs) have also been proposed for engineering intermediate band solar cells. It has been only under this approach that, to our knowledge, actual intermediate band solar cells (QD-IBSC) have been manufactured and has it even been possible to obtain evidence of operation according to the IBSC principles

Our groups have been working on this approach since the year 2000 and we take the opportunity of this paper to compile some of the elements of design and analysis that have guided our approach and that remain still unpublished.

\section{Field damping layer design}

Under the QD approach, the IB arises from the energy levels of the electrons confined by the potential well created by the conduction band offset appearing between the barrier and dot materials (Fig. 2). Additional doping, at the rate of one donor per $\mathrm{QD}$, is usually inserted at the barrier region $(\delta$ doping) to half-fill the IB with electrons by modulated doping effect

The InAs/GaAs material system has been our preferred choice for taking to practice the QD-IBSC concept because this is one of the best known material systems for implementing semiconductor QDs. Although not being an optimum material system for implementing the IBSC from the point of view of potential efficiency, this approach has allowed us to quickly implement operating devices in which it has been possible to test the principles of operation of the IBSC. Additional details of the internal structure of the devices used in the experiments can be found elsewhere and will not be repeated here

Fig. 3 shows a typical result comparing the current-voltage characteristic of a QD-IBSC with that of a GaAs reference solar cell made with the same structure, but without QDs. The QDIBSC consisted of 10 layers of QDs. As it can be observed, photocurrent in the QD-IBSC seems to be the same than the one obtained for the GaAs reference cell. Moreover, voltage is degraded, contrary to what it appears it would be expected.

The problem of the voltage degradation will be discussed in detail in the next section. Concerning the photocurrent, 


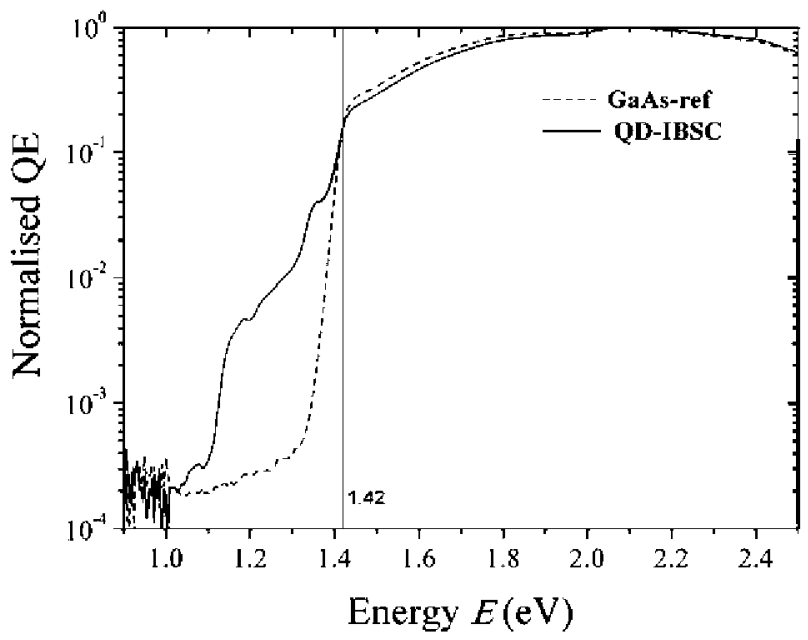

Fig. 4. Normalised quantum efficiency ( $300 \mathrm{~K}$ ) of a QD-IBSC compared with that of the GaAs reference cell.

quantum efficiency measurements reveal, however, that not only below bandgap energy photons are contributing to the production of photocurrent (Fig. 4) but also, that the two below bandgap photon absorption via the intermediate band process described above is taking place [17]. The problem arising is that the absorption of light provided by the dots, although sufficient to demonstrate the intermediate band effect, is too weak as to contribute significantly to enhance the photocurrent of the QDIBSC over that of the GaAs reference.

To enhance significantly the photocurrent implies, for example, to increase the absorption by increasing the number of QD layers. However, when using InAs/GaAs material system for implementing the dots, we have concluded that it is very difficult to increase the number of QD layers beyond 10. This is caused by the fact that the InAs/GaAs is a strained system and dislocations appear when a number of layers beyond this number are attempted to be grown. The bandgap diagram of a QD-IBSC with 10 layers looks more as illustrated in Fig. 5 rather than as it was in Fig. 2, which implies that QDs are not located in a flatband potential region. In this situation it is expected that most of the dot layers cannot serve to the purpose of both being able to

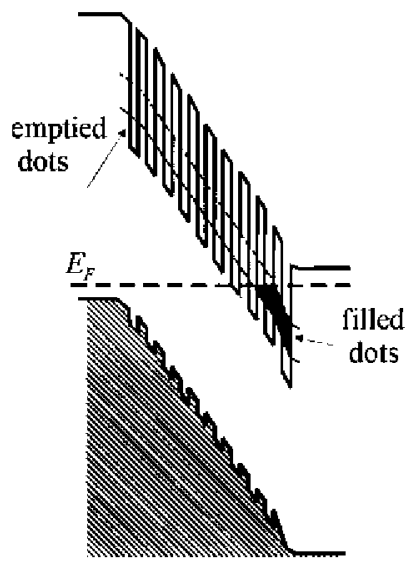

Fig. 5. Simplified bandgap diagram of a QD-IBSC with the QD layers fully immersed in the depletion region

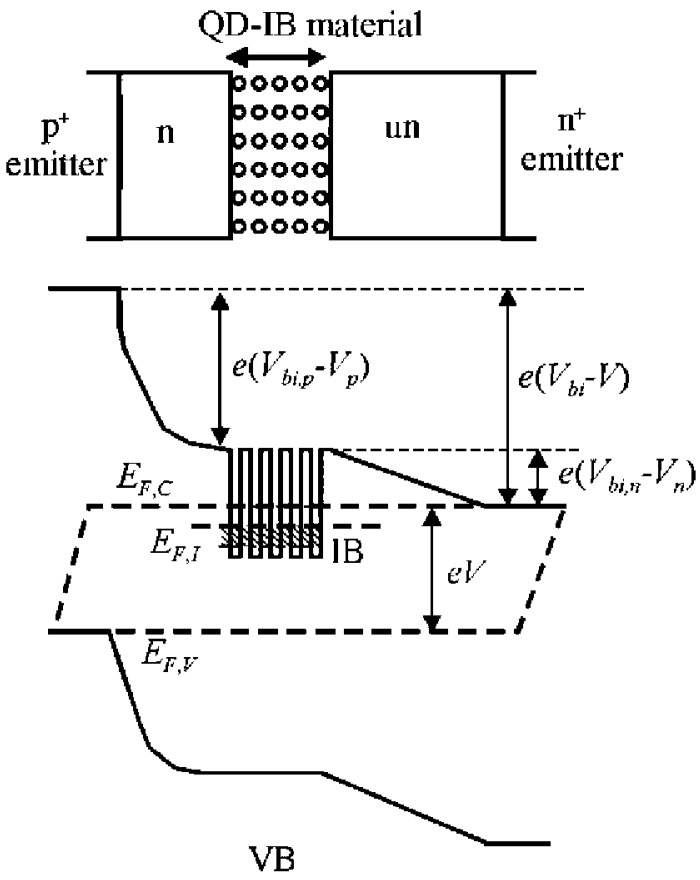

Fig. 6. Location of the field damping layers in the quantum-dot intermediate band solar cell. The built-in potentials are also indicated.

receive electrons form the $\mathrm{VB}$ and to supply them to the $\mathrm{CB}$ because they are either empty (region close to the $p$ emitter) or filled with electrons (region close to the $\mathrm{n}$ emitter).

To make the most of this limited number of layers that can be grown, we devised the concept of field damping layers. These layers consist of an $n$ type layer that is inserted in-between the $p$ emitter and the QD region and an undoped layer that is inserted after the QD region and before the $\mathrm{n}$ type emitter, as indicated in Fig. 6 .

The purpose of the $\mathbf{n}$ type layer located after the $\mathbf{p}$ emitter is to sustain most of the junction build-in potential so that the dots can be driven to a flat-band potential region. The thickness, $W_{\mathrm{n}}$, and doping, $N_{\mathrm{n}}$, of this layer can be calculated using the conventional full depletion approximation for a pn junction

Under this approach, and considering that the $p$ emitter region is much more highly doped than the $\mathrm{n}$ field damping layer,

$W_{\mathrm{n}} \approx\left[\frac{2 \varepsilon_{\mathrm{S}}\left(V_{\mathrm{bi}, \mathrm{p}}-V_{\mathrm{p}}\right)}{e N_{\mathrm{n}}}\right]^{1 / 2}$

where $\varepsilon_{\mathrm{S}}$ is the permittivity of the semiconductor and $V_{\mathrm{bi}, \mathrm{p}}-V_{\mathrm{p}}$ is the potential drop at the $\mathrm{p}$ emitter $/ \mathrm{n}$ field damping layer junction (Fig. 6). Its value can be estimated from:

$V_{\mathrm{bip}}-V_{\mathrm{p}}=V_{b i}-V-\left(V_{\mathrm{bi}, \mathrm{n}}-V_{\mathrm{n}}\right)$

where $V_{\mathrm{bi}}$ is the built-in potential corresponding to the $\mathrm{p}$ emitter/ $\mathrm{n}$ emitter junction. For highly doped emitters, $V_{\mathrm{bi}} \approx 1.4 \mathrm{eV}$. In Eq. (2), $V$ is the applied voltage at which the achievement of the flat-band condition is desired. This voltage should be chosen as that corresponding to the maximum operation point of the solar cell but this can only be known realistically after the cell is 
(a)

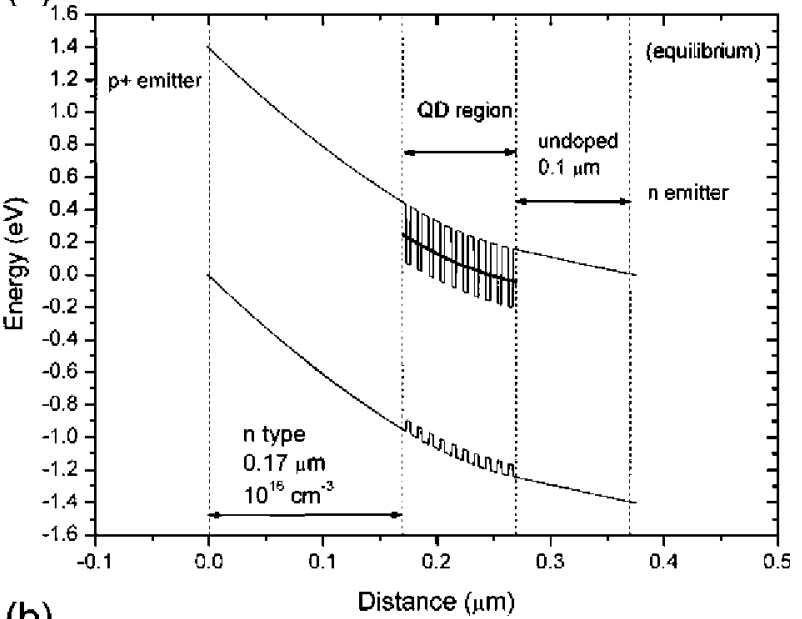

(b)

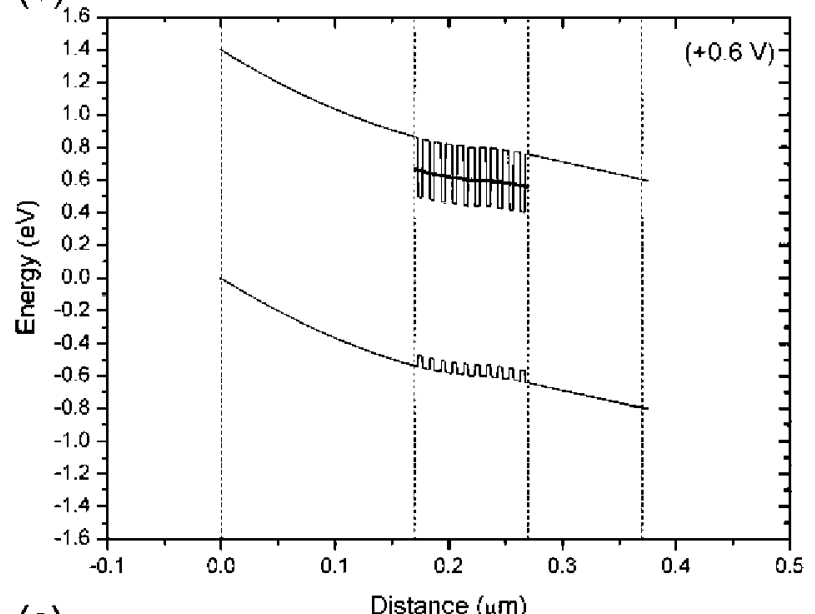

(c)

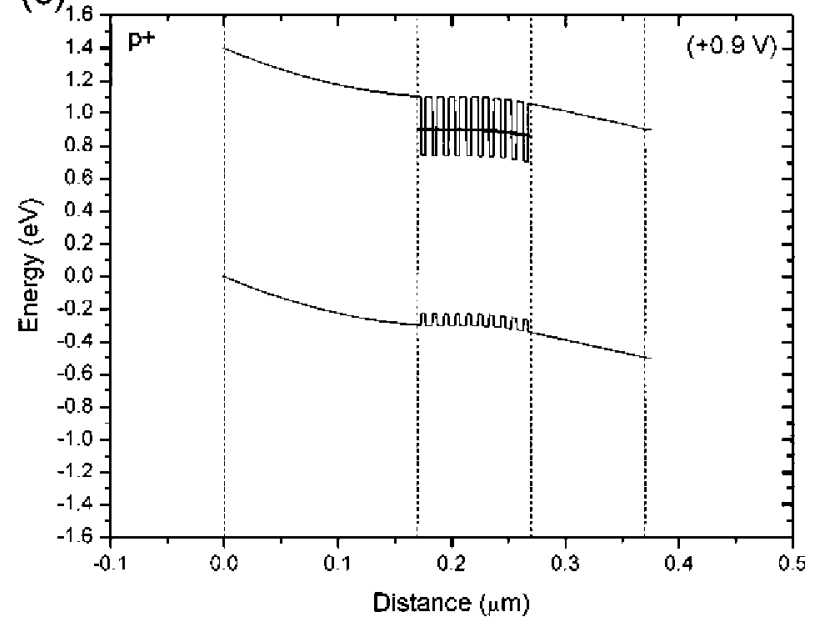

Fig. 7. Simplified bandgap diagram of the space region including field damping layers for several applied voltages.

manufactured. Hence, in order to be on the safe side, we chose $V=1.0 \mathrm{~V}$. Finally, $V_{\mathrm{bi}, \mathrm{p}}-V_{\mathrm{p}}$ is the potential drop at the undoped/ $\mathrm{n}$ emitter junction that we have estimated in $0.2 \mathrm{~V}$. Formally speaking, any pair $\left(N_{\mathrm{n}}, W_{\mathrm{n}}\right)$ satisfying Eq. (1) would suit our needs. However a value too high for $N_{\mathrm{n}}$ (for example in the range the emitter doping) could render invalid the approximations above while a value too low could lead to an excessive thickness that would require, for example, an unnecessarily high time of sample growth. With this considerations in mind, we have finally chosen $N_{\mathrm{n}}=10^{16} \mathrm{~cm}^{-3}$ for our experiments what finally lead to $W_{\mathrm{n}}=0.17 \mu \mathrm{m}$. Fig. 7 shows, for this choice and to scale, our modelling of the bandgap diagram at the space charge region for different applied voltages.

The purpose of the undoped layer is to prevent tunnelling from the $\mathrm{n}$ emitter conduction band into the IB. This tunnelling must be avoided in order to prevent short-circuiting the $\mathrm{CB}$ and IB quasi-Fermi levels situation that would make difficult the required split between these two quasi-Fermi levels. Its thickness is set to $0.1 \mu \mathrm{m}$ in our designs.

\section{Circuit model and voltage loss}

In this section we will set the basis of the elements that allow discussing the voltage loss observed in the QD-IBSC as compared to the GaAs reference cell that was anticipated above.

In the first place, it must be said that the comparison of the output voltage of a QD-IBSC with that of a reference cell without QDs is not a fair comparison. The reason is that since InAs/GaAs QDs are grown in the Stranski-Krastanov growth mode, this causes the appearance of a wetting layer, that is, a quantum well underneath each quantum-dot layer. Quantum wells always introduce one energy level below the conduction band, no matter how thin they are. An additional problem is that this level is not separated from the CB by a true zero density of states and, therefore, forms a continuum with it. On the other hand, it is expected that a valence band offset exists also confining holes in the VB. However, since the hole effective masses are much higher than the electron effective mass, confined energy levels in the VB are more closely spaced than their counterparts in the $\mathrm{CB}$ and, in practice, they are likely to form a quasi-continuum of states with the VB. When this effect is considered together with the gap shrinkage in the CB due to the wetting layer, the total bandgap of the InAs/GaAs quantum-dot system is reduced to about $1.3 \mathrm{eV}$ and sometimes, even less (Fig. 8). Therefore, since the output voltage of a

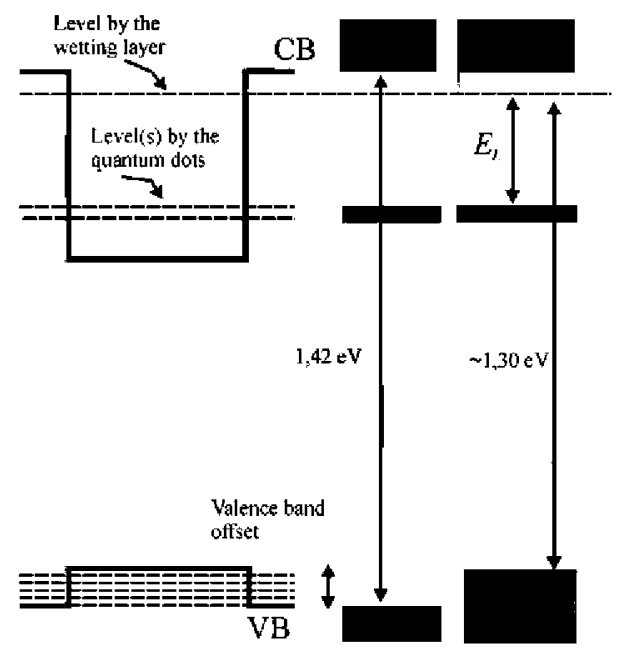

Fig. 8. Illustration of the reduction of the total bandgap in a quantum-dot solar cell as a consequence of the existence of a wetting layer and valence band offset. 
(a)

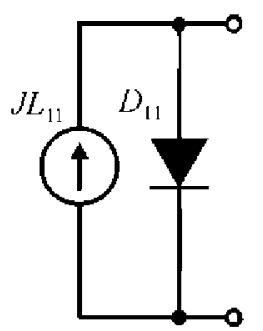

(b)
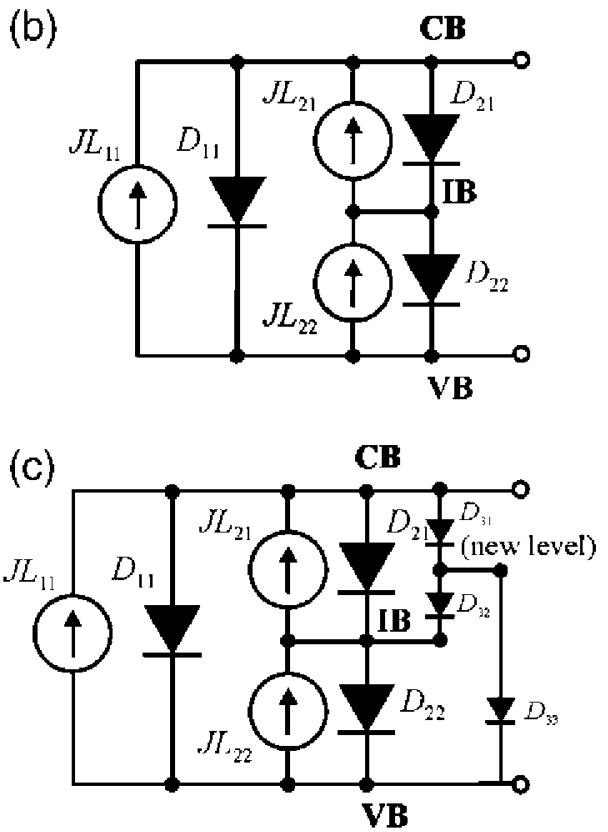

Fig. 9. (a) Simplified equivalent circuit of a single gap solar cell. (b) Simplified equivalent circuit of an intermediate band solar cell. (c) Simplified equivalent circuit of an intermediate band solar cell with additional level appearing inbetween the IB and the VB. This new level is assumed not to contribute to the photogeneration of current.

solar cell cannot exceed its total bandgap, this implies that the output voltage of an InAs/GaAs QD-IBSC should not be straight forward compared to that of a GaAs reference cell $(1.42 \mathrm{eV})$.

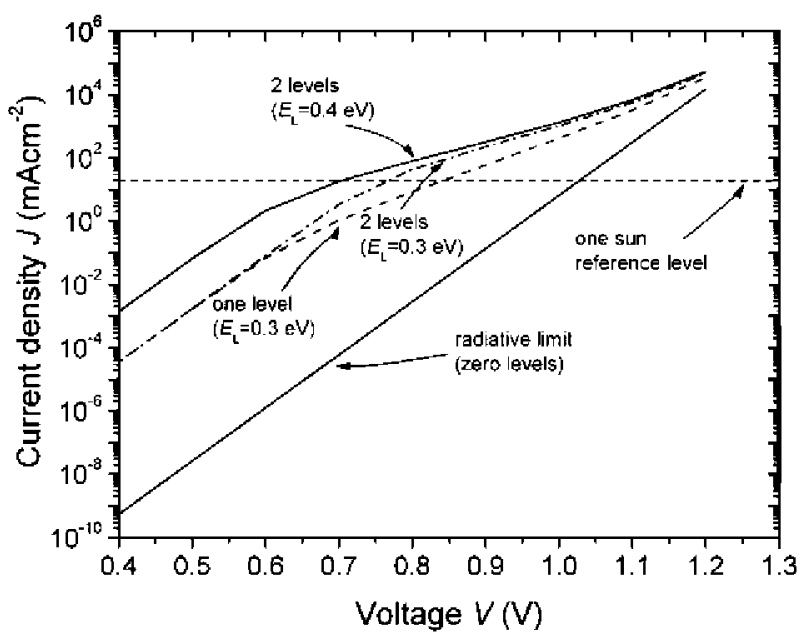

Fig. 10. Analysis of the changes produced in the current-voltage characteristic of an IBSC as several levels are introduced in the energy bandgap.
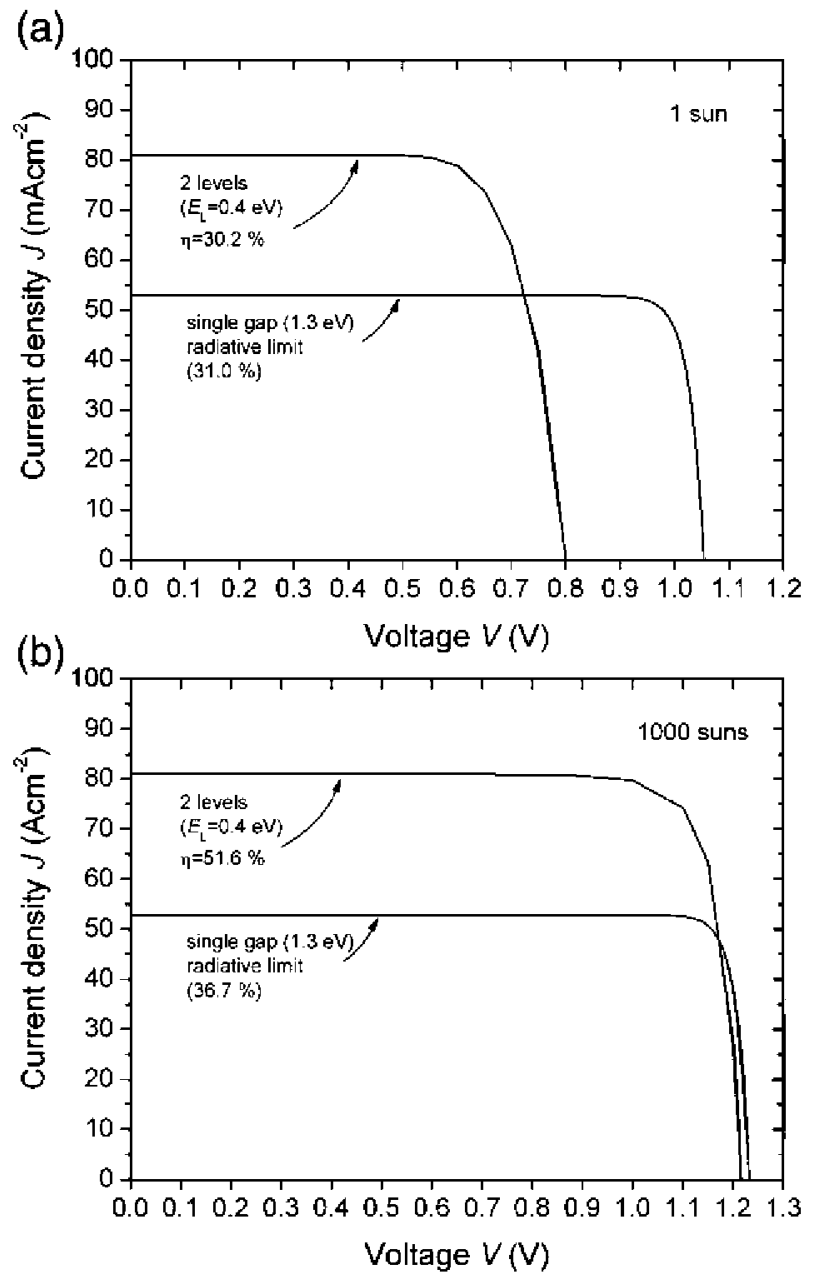

Fig. 11. (a) Current-voltage characteristic at one sun of an ideal solar cell (bandgap $1.3 \mathrm{eV}$ ) operating in the radiative limit and an intermediate band solar cell with an extra level, not contributing to photocurrent, introduced in-between the conduction and intermediate band. (b) The same cells operated at 1000 suns. Black body illumination from a source at $6000 \mathrm{~K}$ has been assumed in all cases Cells are at $300 \mathrm{~K}$.

Once this issue has been clarified, it will be convenient for our discussion to recall the basic simplified circuit of a single gap solar cell (Fig. 9a). Neglecting series and shunt resistance effects, that are not relevant now, the model is constituted by the diode $D_{11}$ and the photocurrent generator $J L_{11}$ only. As it is known, $J_{11}$ counts for the current generated in the cell by the external illumination (strictly speaking, external illumination above thermal equilibrium) and $D_{11}$ counts for the carrier recombination in the cell.

For later reference, we have plotted in Fig. 10 the dark current characteristic that would correspond to this cell (it is labelled as "radiative limit-zero levels") and in Fig. 11 its characteristic under illumination corresponding to 1 sun and 1000 suns. For these calculations we have assumed the cell, with a bandgap of $1.3 \mathrm{eV}$, operating in the radiative limit and the sun assumed as a black body at $6000 \mathrm{~K}$.

When the intermediate band is created within the bandgap, the new set of energy levels opens, in general, a new path for recombination. This new path for carrier recombination is 
modelled in the circuit by two new diodes, $D_{21}$ and $D_{22}$, that connect the $\mathrm{CB}$ with the $\mathrm{IB}$ and the $\mathrm{IB}$ with the $\mathrm{VB}$ respectively (Fig. 9b). However, according to our theory, the IB also opens the path for the absorption of below bandgap energy photons and subsequent production of photocurrent. This photocurrent is modelled by the current generators $J L_{21}$ and $J L_{22}$.

Fig. 10 plots the dark current-voltage characteristic of this cell (the curve is labelled as "one level, $E_{\mathrm{L}}=0.3 \mathrm{eV}$ "). The IB has been assumed located at $0.3 \mathrm{eV}$ below the CB. For making this plot, some hypotheses, that will be described later, have been done about the current going through $D_{21}$ and $D_{22}$ but they are not relevant now to the discussion that follows. What is relevant is to observe that, as expected, at the current reference level set by illumination at one sun (approximately $20 \mathrm{~mA}$ $\mathrm{cm}^{-2}$ ) the voltage is degraded when compared with the dark current-voltage characteristic calculated for the radiative limit. However, it is also observed that, if the illumination level would be raised several orders of magnitude (for example by the use of concentrated light), the output voltage would tend to recover that obtained in the radiative limit. This is a physical consequence of the fact that at high current densities, recombination through diode $D_{11}$, and not through diodes $D_{12}$ and $D_{22}$, become dominant.

Furthermore, in particular when using the QD approach, additional energy levels can appear in-between the IB and the CB. In this case, these levels are originated when, by increasing the dot size, the energy of the fundamental level is shifted towards lower values in the potential well. At first sight, the appearance of these additional energy levels might even look beneficial since they can lead to the formation of additional intermediate bands and could assist the absorption of extra photons. Although this is not completely discounted, it is more likely that the additional level(s) will not be useful for photon absorption due to the difficulty in creating more than one band half-filled with electrons. On the contrary, they may act like "midgap" non-radiative recombination centres for transitions between the CB and the IB.

To model the appearance of this extra energy level (called here "second level" and that will be assumed to be located at the middle of the bandgap $E_{\mathrm{L}}$, where it is most effective for recombination) we have added diodes $D_{31}, D_{32}$ and $D_{33}$ to the circuit model (Fig. 9c) so that diode $D_{31}$ models recombination from the $\mathrm{CB}$ to the second level, diode $D_{32}$ models recombination from the second level to the IB and diode $D_{33}$ models recombination from the second level to the valence band. Notice that no current generators have been associated to these diodes since it has been assumed that they do not contribute to photocurrent. In addition, a new quasi-Fermi level is also associated to this level.

The presence of diodes $D_{3 X}$ modifies again the dark currentvoltage characteristic of the cell as illustrated in Fig. 10 (curve labelled "2 levels, $E_{\mathrm{L}}=0.3 \mathrm{eV}$ "). As expected, the recombination is increased but, again, the influence of the second level in the curve is lost as the current density increases approaching again the plot in which recombination through diode $D_{11}$ (radiative limit) is dominant.

As an additional case of study we have also considered the case in which the level $E_{\mathrm{L}}$ is shifted towards $0.4 \mathrm{eV}$. From the perspective of the IBSC theory, $0.4 \mathrm{eV}$ is closer to the optimum value than the $0.3 \mathrm{eV}$ case previously considered. If we repeat the calculations above, including the presence of a second level in-between the IB and the CB, the dark current characteristic becomes the highest of all (curve labelled "2 levels, $E_{\mathrm{L}}=0.4 \mathrm{eV}$ " in Fig. 10). This increment could be regarded as a logical consequence of the fact of approaching the IB towards the midgap, where recombination is more effective and, from this point of view, it will be useful to our discussion as a worst case analysis. It is important to notice that, again, at high current densities the impact on the curve due to this shift decreases. This effect is better illustrated by comparing the current-voltage characteristic of this cell at one sun and 1000 suns with that of the single gap solar cell operated in the radiative limit (Fig. 11). As it can be seen, at one sun, the IBSC cell (plot labelled "2 levels, $E_{\mathrm{L}}=0.4 \mathrm{eV}$ ) exhibits a poorer efficiency than its single gap counterpart. This is because, although the IBSC increases its short-circuit current due to the absorption of below bandgap energy photons, this increment does not suffice to overcome the voltage loss. However at 1000 suns the situation is radically different and the efficiency of the IBSC exceeds clearly that of the single gap solar cell even when the latest has been assumed to operate in the radiative limit. This is a consequence of the fact that, at high current densities, the IBSC has been able to recover its capacity to provide high open-circuit voltage and also to the fact that, at high current densities, the value of the optimum bandgap $E_{\mathrm{G}}$ decreases.

We end this section by clarifying the current models assumed for the diodes and current generators used in the examples above. By now it should be clear, nevertheless, that the tendency illustrated (recovering the open-circuit voltage as the current density increases) is general and not fundamentally dependent on the diode model used.

As mentioned, the cells have been assumed to be illuminated by blackbody radiation at temperature $T_{\mathrm{S}}=6000 \mathrm{~K}$. According to the basic IBSC model, this implies that the current supplied by the generators is given by:

$$
\begin{aligned}
& \mathrm{JL}_{11}=X \pi \operatorname{sen}^{2} \theta_{\mathrm{S}}\left[F\left(E_{\mathrm{G}}, \infty, 0, T_{\mathrm{S}}\right)-F\left(E_{\mathrm{G}}, \infty, 0, T_{\mathrm{C}}\right)\right] \\
& \mathrm{JL}_{21}=X \pi \operatorname{sen}^{2} \theta_{\mathrm{S}}\left[F\left(E_{\mathrm{L}}, E_{\mathrm{H}}, 0, T_{\mathrm{S}}\right)-F\left(E_{\mathrm{L}}, E_{\mathrm{H}}, 0, T_{\mathrm{C}}\right)\right] \\
& \mathrm{JL}_{22}=X \pi \operatorname{sen}^{2} \theta_{\mathrm{S}}\left[F\left(E_{\mathrm{H}}, E_{\mathrm{G}}, 0, T_{\mathrm{S}}\right)-F\left(E_{\mathrm{H}}, E_{\mathrm{G}}, 0, T_{\mathrm{C}}\right)\right]
\end{aligned}
$$

where $X$ is the light concentration, $\theta_{\mathrm{S}}$ is the sun's semi-angle of vision $\left(1 / \operatorname{sen}^{2} \theta_{\mathrm{S}} \approx 46,050, T_{\mathrm{C}}\right.$ is the cell temperature $(300 \mathrm{~K})$ and

$F\left(E_{\mathrm{A}}, E_{\mathrm{B}}, \mu, T\right)=\frac{2}{h^{3} c^{2}} \int_{E_{\mathrm{A}}}^{E_{\mathrm{B}}} \frac{2 \varepsilon^{2}}{\exp \left(\frac{\varepsilon-\mu}{k T}\right)-1} d \varepsilon$

with $h$ being the Planck's constant and $c$ the speed of light in vacuum. 
The current-voltage relationship for the diodes $D_{X Y}$ appearing in the circuit models, has been assumed to be given by an equation of the type

$J_{X Y} \approx J_{0, X Y}\left(\exp \frac{e V_{X Y}}{m k T}-1\right)$

where $J_{X Y}$ and $V_{X Y}$ are respectively the current and voltage across the diode. The factor "- 1 ", counting for carrier thermal generation, should not be lightly disregarded, in particular when small bandgaps are involved (because then the value of $J_{0, X Y}$ is large) and/or the circuit is used to model small currents. The diode factor, $m$, has been set here equal to one. The diode reverse saturation currents have been assumed to be given by:

$J_{0, X Y}=\frac{2 \pi}{h^{3} c^{2}} \int_{E_{X Y}}^{\infty} \varepsilon^{2} \exp \left(\frac{-\varepsilon}{k T_{\mathrm{C}}}\right) d \varepsilon$

where $E_{X Y}$ is the bandgap associated to the bands in-between the diode is inserted $\left(E_{11} \equiv E_{\mathrm{L}} ; E_{21} \equiv E_{L}, E_{22} \equiv E_{\mathrm{H}}, E_{31} \equiv E_{\mathrm{L}} / 2\right.$; $\left.E_{32} \equiv E_{\mathrm{L}} / 2 ; E_{33} \equiv E_{\mathrm{H}}+E_{32}\right)$. The physical basis for the recombination model set by Eq. (8) have been: (a) recombination between bands is assumed to be radiative; (b) the quasi-Fermi level split between bands is lower than the bandgap (nondegeneracy) and (c) as recombination is concerned, diodes emit photons with energy from $E_{X Y}$ to infinity.

\section{Conclusions}

We have described the application of field damping layers to allocate quantum dots in a flat-band potential region in order to maximize the absorption of light when a limited number of QD layers are available by the technology and also to prevent tunnelling from the $\mathbf{n}$ emitter into the intermediate band. It is believed that this design has allowed showing the absorption of two below bandgap energy photons to produce one electronhole pair (described in another paper [17]) but is still insufficient to increase significantly the contribution to the photocurrent provided by the dots. This contribution will be essential to overcome the voltage loss induced by the presence of additional energy levels within the bandgap.

On the other hand, the voltage loss observed in QD-IBSCs is expected to decrease if concentrated light is used. This is due to the fact that as current density increases, the impact on recombination due to intermediate band(s) diminishes and recombination from the conduction band to the valence band becomes dominant.

\section{References}

A. Luque, A. Martí, Phys. Rev. lett. 78/26 (1997) 5014

A. Luque, A. Martí, Prog. Phycol. Res. Appl. 9/2 (73) (2001).

A. Luque, A. Martí, E. Antolín, C. Tablero, Physica B 382 (2006) 320.

A. Luque, A. Martí, L. Cuadra, IEEE Trans. Electron. Devices 48/9 (2001) 2118.

A. Luque, A. Martí, L. Cuadra, IEEE Trans. Electron. Devices 50/2 (2003) 447.

P. Palacios, J.J. Fernandez, K. Sanchez, J.C. Conesa, P. Wahnon, Phys. Rev., B, Condens. Matter Mater. Phys. 73/8 (2006) 085206.

P. Palacios, K. Sanchez, J.C. Conesa, P. Wahnon, Phys. Status Solidi, A Appl. Mater. Sic. 203/6 (2006) 1395.

C. Tablero, Phys. Rev., B 74/19 (2006).

C. Tablero, Comput. Mater. Sci. $37 / 4$ (2006) 483.

C. Tablero, Sol. Energy Mater. Sol. Cells 90/5 (2006) 588.

K.M. Yu, W. Walukiewicz, J. Wu, W. Shan, J.W. Beeman, M.A. Scarpulla, O.D. Dubon, P. Becla, Phys. Rev. Lett. 91/24 (2003) 246403.

K.M. Yu, W. Walukiewicz, J.W. Ager, D. Bour, R. Farshchi, O.D. Dubon, S.X. Li, I.D. Sharp, E.E. Haller, Appl. Phys. Lett. 88/9 (2006) 092110.

W. Shan, W. Walukiewicz, J.W. Ager, E.E. Haller, J.F. Geisz, D.J Friedman, J.M. Olson, S.R. Kurtz, Phys. Rev. Lett. 82/6 (1999) 1221.

A. Martí, L. Cuadra, A. Luque, Proc. of the 28th IEEE Photovoltaics Specialists Conference, New York, 2000.

A. Luque, A. Martí, C. Stanley, N. Lopez, L. Cuadra, D. Zhou, J.L. Pearson, A. McKee, J. Appl. Phys. 96/1 (2004) 903.

A. Luque, A. Martí, N. Lopez, E. Antolin, E. Canovas, C. Stanley, C. Farmer, L.J. Caballero, L. Cuadra, J.L. Balenzategui, Appl. Phys. Lett. 87/ 8 (2005) 083505

A. Martí, E. Antolín, C.R. Stanley, C.D. Farmer, N. López, P. Diaz, E. Canovas, P.G. Linares, A. Luque, Phys. Rev. Lett. 97/24 (2006) 247701.

A. Martí, L. Cuadra, A. Luque, in: A. Martí, A. Luque (Eds.), Next Generation Photovoltaics: High Efficiency through Full Spectrum Utilization, Institute of Physics Publishing, Bristol, 2003, p. 140.

A. Martí, C.R. Stanley, A. Luque, in: T. Soga (Ed.), Nanostructured Materials for Solar Energy Conversion, Elsevier, 2006.

A. Martí, L. Cuadra, A. Luque, IEEE Trans. Electron. Devices 48/10 (2001) 2394.

A. Luque, A. Martí, N. López, E. Antolín, E. Cánovas, C.R. Stanley,

C. Farmer, P. Díaz, J. Appl. Phys. 99/1 (2006) 094503.

A. Martí, N. López, E. Antolín, E. Cánovas, C. Stanley, C. Farmer, L. Cuadra, A. Luque, Thin Solid Films 511-512 (2006) 638.

G.W. Neudeck, The pn Junction Diode, Addison-Wesley, Reading, 1989. M. Sugawara, Self-assembled InGaAs/GaAs Quantum Dots, Academic Press, 1999.

C. Weisbuch, B. Vinter, Quantum Semiconductor Structures, Academic Press, San Diego, 1991.

W. Shockley, H.J. Queisser, J. Appl. Phys. 32/3 (1961) 510.

G.L. Araújo, A. Martí, Sol. Energy Mater. Sol. Cells 33/2 (1994) 213. 